\title{
Modelling Open Kinematic Chains through General Matrix Movements
}

\author{
M. Granja, A. Chang \\ Escuela Politécnica Nacional \\ Ladrón de Guevara, E11-253 Quito, Ecuador \\ mario.granja@epn.edu.ec; nikolas.chang@epn.edu.ec
}

\begin{abstract}
In this work, an innovative method for modelling open kinematic chains in robots is presented, in order to decrease the difficulty level at the moment of assign coordinates systems and obtaining the Denavit-Hartenberg parameters. This method is based in the post-multiplication matrix method, and the fundamental principle of the standard and modified Denavit-Hartenberg's method presented sixty years ago. The method begins of the knowledge of the rest configuration of the robot, to obtain the degrees of freedom of the robot upwardly. To obtain the transformation of coordinates equations, we just has to apply the knowledge of the method to transform coordinates systems geometrically according to general methods. The Continuous General Movement leads to obtain the transformation coordinates matrix, rotations and translations that allow to know the position of the final effector in the robot based in the variables of the robot and the fixed coordinates system. Unlike other methods, it has not have to make some strange movements or assumptions to reach the answer, the Continuous General Movement works in the same direction that the robot moves itself; to reach the final effector takes less parameters and steps to follow, reaching a similar answer as the original method of Denavit-Hartenberg and it involves simple matrix operations and is easily programmable.
\end{abstract}

Keywords: Continuous General Movement, Denavit-Hartenberg, post-multiplication matrix, robot.

\section{Introduction}

Denavit and Hartenberg (Denavit and Hartenberg, 1955) developed a method to obtain the kinematic model of kinematic chains, stablishing how the coordinated systems must be associated to each individual link of the kinematic chain, in order to obtain of the transformation coordinates matrices from the system ${ }^{\mathrm{i}-1} \mathrm{~A}_{\mathrm{i}}$, between the system formed by the link $i-1$ and the link $i$. The multiplication of those matrices allows to obtain an expression to change of coordinates matrices between the associate coordinate systems, that involves the degrees of freedom (DOF) of the robot, in order to know the expressions that defines the positions and orientation of the end of the robot in function of the articulate coordinates (qi). These relations are the Direct Kinematic Model of the robot.

Obtaining this model is essential to confront most of the problem associated with control and programming of manipulators, walkers or humanoid robots, by the matrix ${ }^{\mathrm{i}-1} \mathrm{~A}_{\mathrm{i}}$, which is function of the four Denavit-Hartenberg parameters $(\theta, d, a, \alpha)$ associated to four consecutive movements (rotation and translation in $\vec{Z}$, called $\mathrm{Z}$ screw, followed by a translation and rotation in $\vec{x}$, called $\mathrm{X}$ screw). To make this possible, is precise to associate each link to a coordinates system, following specific rules stablished by Denavit and Hartenberg.

Many works has been developed during the last century like, Pieper (Pieper, 1968) used the matrix representation of the relation between 2 links connected by one joint, to get the direct kinematic model of the robots. The dual numbers (Veldkamp, 1976) used in the matrix context, matrices (3x3), being applicable to the solution of the kinematic robot model (McCarthy, 1986), (Jin and Xiaorong, 2010). Quaternions, allows the representation of rotations in a more compact format, but the rotation matrices by themselves cannot represent translations. (Perez and McCarthy, 2004). Exponential Product (PoE for its acronym in English) (Brockett, 1984), (Murray et al., 1994), uses the exponential series representation of a matrices to represent screw displacements. Displacement matrix method (MD) (Barrientos-Álvares, et al., 2012), by expanding the Rodriguez rotation formula, to translations along axis that not pass through the origin of coordinates.

In any case, the popularity of DH method has eclipsed so many alternatives in the field of basis robotics, possibly because of the less widely algebraic basis through the use of homogeneous transformation matrices to represent the base changes without noticeable advantages. The DH method is conceptually simple; the user just has to apply some basic knowledge of linear algebra. But its use often has some difficulty due to the spatial selection complexity linked to the location of the coordinate systems associated with each link, being the main reason for some books and works mistakes. 
In this work, a new methodology is presented, showing the method to obtain the direct kinematic model of robots in open kinematic chains, with only basic knowledge of lineal algebra and the matrix post-multiplication. The final expression obtained is equivalent to the one obtained with the DH method, without having to precisely achieve the assignation of coordinate systems, or making strange assumption to fit the DH method in spatial open kinematic chains. Systemizing and simplifying the method to obtain the kinematic model of the robots.

The proposed method use the Continuous General Movement (MGC) that involves the assignation of coordinates systems and movements through the robot in the same direction of the movements, in the same sequence to reach them.

Finally this method simplifies the fact that in the DH method exist 2 forms, the standard method, and assignment of coordinated systems in each link, and the modified method, assignment of coordinated systems in each joint. Is hopped, that after the knowledge of this new method and its demonstration, this constitutes a competitive and even preferred method to modeling open kinematic chains. The objective to show and evaluate the difference between this new methodology and DH standard method is searched.

The structure of the present article begins with an explanations of the terms involved, performing representations in 2 dimensions and 3 dimensions. In section 3 the MGC method is presented, in section 4 a direct application of the method against the DH method is shown. Allows to see how the MGC with a simplified methodology, obtains an equivalent results as the DH method, using the representation of a well know robot, the Stanford Robot (schematic representation), and showing the readers that this method is compatible with robots with complicated kinematics. It is supposed that the reader is familiarized with Denavit-Hartenberg method (Ollero. 2001), (Spong-Hutchinson, et al., 2004), (Barrientos et al. 2007).

\section{Theoretical Framework}

\subsection{Degrees of freedom of a mechanism (DOF)}

DOF of a link or a rigid body, is defined like the number of independent movement that it has. In the two-dimension plane, there is a tree DOF. The link could be translated along the $X$ and $Y$ axis, and turn around a perpendicular axis to the $X Y$ plane. In the other hand, a spatial mechanism has six DOF that consist in tree displacements along the $\mathrm{X}, \mathrm{Y}$ and $\mathrm{Z}$ axis, and tree rotation movements around de $\mathrm{X}, \mathrm{Y}$ and $\mathrm{Z}$ axis respectively. These movements are shown in the figure 1
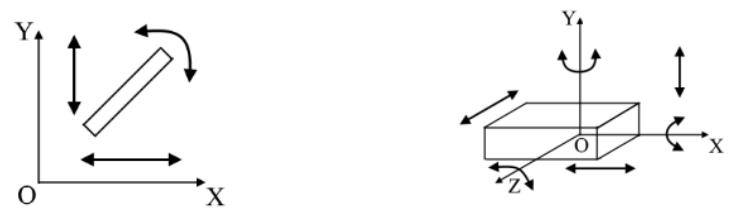

Fig. 1: Degrees of freedom of a free link, left: planar; right: spatial.

\subsubsection{Kinematic Restrictions}

Two or more links or rigid bodies in space are denominated collectively one system. The independent movements of the links could be restricted with kinematic restrictions; these restrictions are limitations between the rigid bodies that decrease the number of DOF of the system. The term used to describe this restrictions, is "kinematic pairs" and they are divided in two categories, the superior and inferior pairs, depending how the contact is. The type of pairs used in all the manipulator robots, are the inferior pairs.

\subsubsection{Inferior pairs}

There are two types of inferior pair in planar mechanism: the revolution pairs and the prismatic pairs, shown in the figure 2. A rigid body in the plane just has 3 independent movements, two of translation and one rotation; consequently, introducing a revolution or prismatic pair, will reduce two DOF in the system.
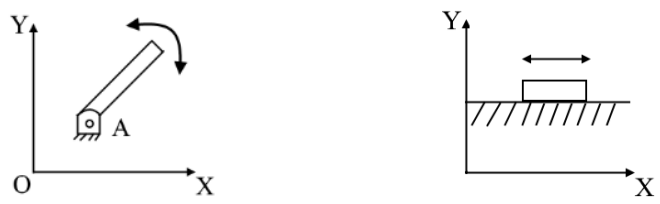
Fig. 2: Kinematic restrictions in planar mechanisms, left: revolution; right: prismatic.

Meanwhile in the spatial mechanism, there are six different types of inferior pairs, these are: cylindrical pair, revolution pair, prismatic pair and screw pair, these representations are shown in the figure 3.
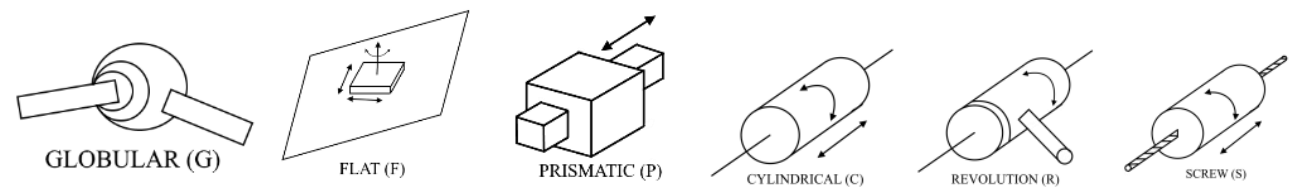

Fig. 3: Kinematic restrictions in spatial mechanisms.

\subsubsection{Calculation of the DOF}

A free link in the plane has 3 degrees of freedom. The kinematic pairs are restrictions that reduce the DOF mechanism, there are 3 types of planar mechanism, superior pairs that reduce two DOF or superior pairs that reduce one DOF. For establish the relationship between these restrictions and free links, Grübler developed an equation, equation (1), to describe this phenomena and calculate the number or DOF in a system composed by any number of joints or links.

$$
\mathrm{DOF}=3(\mathrm{n}-1)-2 j_{1}-j_{2}
$$

Where $\mathrm{n}$ is the number of links, including the fixed one, $j_{1}$ is the number of inferior pairs and $j_{2}$ is the number of superior pairs. But to represent the relationship in spatial mechanism, another equation based in the same criteria was developed by Kutzbach, Equation (2). This is the mobility of the mechanism that represents the number of parameters that should be controlled independently to guide the device to a particular position.

$$
\text { DOF }=6(\mathrm{n}-1)-5 j_{1}-4 j_{2}-3 j_{3}-2 j_{4}-1 j_{5}
$$

Where $\mathrm{n}$ is the number of links, including the fixed one, $j_{1}$ is the number of joints with $1 \mathrm{DOF}, j_{2}$ is the number of joints with $2 \mathrm{DOF}, j_{3}$ is the number of joints with $3 \mathrm{DOF}, j_{4}$ is the number of joints with $4 \mathrm{DOF}, j_{5}$ is the number of joints with 5 DOF. With the purpose of controlling the mechanism, the number of independent in movements must be equal to the number of degrees of freedom of the mechanism.

\subsection{Coordinate Systems, position and orientation}

The fundamental theory shown in this section would be necessary to determinate the direct and inverse kinematic, the representation used to determinate the model, is based in the equations used to translate or rotate coordinated systems from the fixed system $o_{0} x_{0} y_{0} z_{0}$ to the final effector of the robot in the system $o_{n} x_{n} y_{n} z_{n}$

\subsubsection{Matrices of rotation}

In a rotation, a point $\mathrm{P}$ and two systems with the same origin of coordinates must be considered, because the point will have two referred systems, the fixed system $o, x, y, z$ and the mobile system $o, u, v, w$; $\mathrm{P}$ could be represented by a position vector referred to the two coordinated systems. The unitary vectors of the systems are $\vec{\imath}_{x}, \vec{\jmath}_{y}, \vec{k}_{z}$, and $\vec{l}_{u}, \vec{\jmath}_{v}, \vec{k}_{w}$ respectively. The representation of the two coordinated systems and the position vector of the point $\mathrm{P}$ is shown in the figure 5. With this representation, the point $\mathrm{P}$ could be represented by the equations (3) or (4)

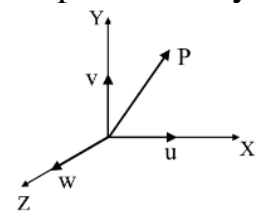

Fig. 5: Fixed and mobile coordinate systems of P. 


$$
\begin{gathered}
\vec{P}_{x, y, z}=P_{x} \vec{\imath}_{x}+P_{y} \vec{\jmath}_{y}+P_{z} \vec{k}_{z} \\
\vec{P}_{u, v, w}=P_{u} \vec{\imath}_{u}+P_{v} \vec{\jmath}_{v}+P_{w} \vec{k}_{w}
\end{gathered}
$$

Using the scalar product in the position vector (3) and the unitary vectors of (4), depending of what coordinate is required, a set of equations arranged in matrix representation according to the algebraic basis is obtained, and knowing that this matrix represents the rotation of the unitary vectors from the fixed system to the mobile system , this matrix is orthonormal. It is represent by equation (5).

$$
R=\left[\begin{array}{l}
p_{x} \\
p_{y} \\
p_{z}
\end{array}\right]=\left[\begin{array}{lll}
\vec{i}_{u} \cdot \vec{i}_{x} & \vec{j}_{v} \cdot \vec{i}_{x} & . \vec{i}_{x} \\
\vec{j}_{v} \cdot \vec{i}_{y} & \vec{j}_{v} \cdot \vec{i}_{y} & \vec{j}_{v} \cdot \vec{i}_{y} \\
\vec{k}_{w} \cdot \vec{i}_{z} & \vec{k}_{w} \cdot \vec{i}_{z} & \vec{k}_{w} \cdot \vec{i}_{z}
\end{array}\right]\left[\begin{array}{c}
p_{u} \\
p_{v} \\
p_{w}
\end{array}\right]
$$

Now, using the matrix equation (5) and the figure 6 in which the rotation around axis $\mathrm{X}, \mathrm{Y}$ and $\mathrm{Z}$ are represented, the rotation matrices around those axis could be determinate.
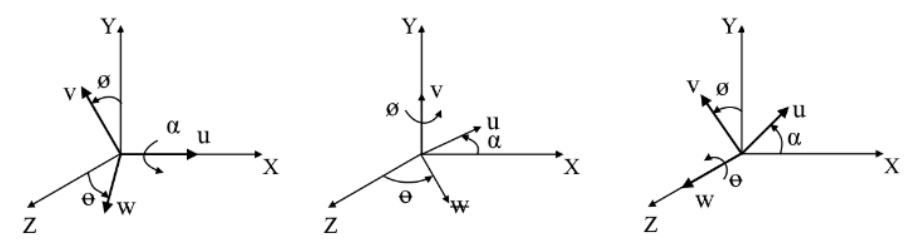

Fig. 6: Rotation of the system $\mathrm{p}, \mathrm{u}, \mathrm{v}, \mathrm{w}$ around $x, \propto, y, \phi z, \theta$

Obtaining the pure rotation matrix of order $3 \times 3$ shown below (Equation (6), (7) and (8)), adding the identity $1=1$ to each matrix, the formulation could be expressed in matrices $4 \times 4$.

$$
\begin{aligned}
R_{(x, \propto)} & =\left[\begin{array}{cccc}
1 & 0 & 0 & 0 \\
0 & \cos \propto & -\operatorname{sen} \propto & 0 \\
0 & \operatorname{sen} \propto & \cos \propto & 0 \\
0 & 0 & 0 & 1
\end{array}\right] \\
R_{(y, \varnothing)} & =\left[\begin{array}{cccc}
\cos \emptyset & 0 & \operatorname{sen} \varnothing & 0 \\
0 & 1 & 0 & 0 \\
-\operatorname{sen} \varnothing & 0 & \cos \varnothing & 0 \\
0 & 0 & 0 & 1
\end{array}\right] \\
R_{(Z, \theta)} & =\left[\begin{array}{cccc}
c \theta & -\operatorname{sen} \theta & 0 & 0 \\
\operatorname{sen} \theta & \cos \theta & 0 & 0 \\
0 & 0 & 1 & 0 \\
0 & 0 & 0 & 1
\end{array}\right]
\end{aligned}
$$

\subsubsection{Matrix of translation}

To translation, a point $\mathrm{P}$, a fixed coordinated system $o, x, y, z$ and a mobile system $o, u, v, w$ that has been translated $\Delta x$ along the axis $x_{1}, \Delta y$ along the axis $y_{1}$, and $\Delta z$ along the axis $z_{1}$. The coordinates of the point $\mathrm{P}$ are known, and it moves with the mobile system. To determinate the equations to relation the system $o, x, y, z$ as function of $o, u, v, w$, the figure 8 will be used and considering the identity $1=1$ we can obtain equations that relation the systems only by geometrical relations. 


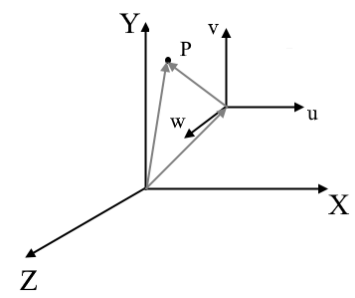

Fig. 8: Coordinates System Representation, Translation.

$$
\left[\begin{array}{c}
p_{x} \\
p_{y} \\
p_{z} \\
1
\end{array}\right]=\left[\begin{array}{llll}
1 & 0 & 0 & d_{x} \\
0 & 1 & 0 & d_{y} \\
0 & 0 & 1 & d_{z} \\
0 & 0 & 0 & 1
\end{array}\right]\left[\begin{array}{c}
p_{u} \\
p_{v} \\
p_{w} \\
1
\end{array}\right]
$$

\subsection{Matrix Post-multiplication and matrix Pre-multiplication}

Post-multiplication: Making movements with mobile system of coordinates, referred to the axis of the last mobile coordinated system, allow us to obtain the transformation coordinates equation between the last coordinates system and the fixed coordinates system, to make this happen the matrix product should be done in the same sequence of the movements. This matrix product is used in the Euler angles, Equation (12) where tree consecutives rotations are made, $\propto$ around the $\mathrm{Z}$ axis, $\beta$ around the $\mathrm{Y}$ axis and $\gamma$ around the $\mathrm{X}$ axis, and this rotations are referred to the last mobile coordinates system.

$$
R_{(\alpha, \beta, \gamma)}=R_{z 1, \alpha} \cdot R_{y 2, \beta} \cdot R_{x 3, \gamma}=\left[\begin{array}{ccc}
c \alpha c \beta & c \alpha s \beta s \gamma-s \alpha c \gamma & c \alpha s \beta c \gamma-s \alpha s \gamma \\
s \alpha c \beta & s \alpha s \beta s \gamma+c \alpha c \gamma & s \alpha s \beta c \gamma-c \alpha s \gamma \\
-s \beta & c \beta s \gamma & c \beta c \gamma
\end{array}\right]
$$

Pre-multiplication: If the movements of the mobile coordinates system is referred to the fixed coordinates system, the matrices has to be multiplied in reverse sequence of the movements, known as matrix pre-multiplication. Matrix RPY (roll, pitch and yaw) is obtained by this method, Equation (13). The sequence of movements are, an angle $\gamma$ around the $\mathrm{X}$ axis (Roll), an angle $\beta$ around the $\mathrm{Y}$ axis (pitch), and a rotation $\alpha$ around the $\mathrm{Z}$ axis (yaw).

$$
R_{(\alpha, \beta, \gamma)}=R_{z 0, \alpha} \cdot R_{y 0, \beta} \cdot R_{x 0, \gamma}=\left[\begin{array}{ccc}
c \alpha c \beta & c \alpha s \beta s \gamma-s \alpha c \gamma & c \alpha s \beta c \gamma-s \alpha s \gamma \\
s \alpha c \beta & s \alpha s \beta s \gamma+c \alpha c \gamma & s \alpha s \beta c \gamma-c \alpha s \gamma \\
-s \beta & c \beta s \gamma & c \beta c \gamma
\end{array}\right]
$$

\section{Continuous General Movement (MGC)}

Once understood the transformation of coordinates matrix for translations and rotations of planar and spatial mechanisms, as well as the matrix pre and post-multiplication. The reader is prepared to apply this knowledge to converge in the continuous general movement method.

Regard the fact that in other methods, the identification of all the parameters is required to developed the first steps, in the proposed method, is just necessary a geometrical representation (Spong-Hutchinson, et al, 2004), of the robot in its initial or referent position. The methodology steps are numbered next:

1. Identify the fixed coordinate system $\left(o_{0} x_{0} y_{0}\right.$ for planar systems, $o_{0} x_{0} y_{0} z_{0}$ for spatial systems)

2. From the fixed coordinate systems, continuously moves the mobile coordinate system until reach the end, following the kinematic chain of the mechanism, from the base to the end.

3. For each individual movement assign a variable depending of the nature of the movement, use $d$ for displacements along $Z$ axis, $\theta$ to turns around $Z$ axis, use $\mathrm{b}$ for displacements along $Y$ axis, $\beta$ to turns around $Y$ axis, use $a$ for displacements along $X$ axis, $\alpha$ to turns around $X$ axis.

4. Apply the matrix post-multiplication concept, multiplying every matrix movement obtained in the point 4 in the same sequence of the movements. 
5. Obtain the matrix representation, to the change of base from the origin $o_{0} x_{0} y_{0} z_{0}$ to the final link $o_{n} x_{n} y_{n} z_{n}$

\section{Application}

Continuing with the integration of the process in one well known robot configuration, the Stanford robot with six degrees of freedom. All the results are susceptible of comparison and in at the end a quantitative comparison between the MGD and DHs will be make. In this case, the coordinates systems and parameters of the DH standard method, as well as the representation in each case.

\subsection{Robot Stanford with DHs}

Figure 9, is a valid representation by using the geometrical representation (Spong-Hutchinson, et al, 2004) of the Stanford robot involving the DHs method to obtain the parameters, with figure 12 the reader could see the coordinates system assignation; by nature the Stanford robot has 6 axis, in consequence it has 6 DOF, the rotation variables to control are $\theta_{i}$, meanwhile the displacement variable is $d_{i}$, everything else is quantified.

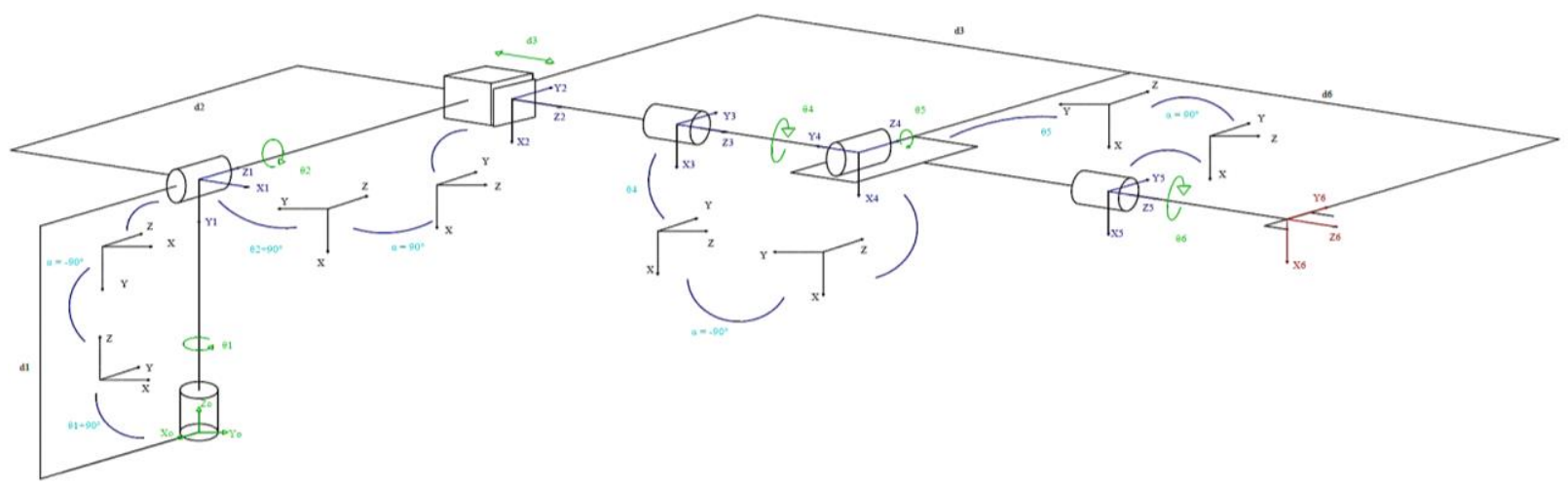

Fig. 9: Movements of the coordinated systems through the Stanford Robot using DHs

Through the representation in figure 9, D-H standard table could be obtained (Table 1), following the sequence of movements stablished for the method, first a screw movement along $\mathrm{Z}$ axis, and following a screw movement along $\mathrm{X}$ axis.

Table 1: DHs parameters of a Stanford robot.

\begin{tabular}{|c|c|c|c|c|}
\hline Link & $\theta_{i}$ (Rot. z Axis $)$ & $d_{i}$ (Displacement z Axis $)$ & $a_{i}$ (Displacement x Axis $)$ & $\alpha_{i}$ (Rot. x Axis) \\
\hline 1 & $\theta_{1}^{*}+90^{\circ}$ & $d_{1}$ & 0 & $-90^{\circ}$ \\
\hline 2 & $\theta_{2}^{*}+90^{\circ}$ & $d_{2}$ & 0 & $90^{\circ}$ \\
\hline 3 & 0 & $d_{3}^{*}$ & 0 & 0 \\
\hline 4 & $\theta_{4}^{*}$ & 0 & 0 & $-90^{\circ}$ \\
\hline 5 & $\theta_{5}^{*}$ & 0 & 0 & $90^{\circ}$ \\
\hline 6 & $\theta_{6}^{*}$ & $d_{6}$ & 0 & 0 \\
\hline
\end{tabular}

$$
\begin{aligned}
{ }^{i-1} A_{i}= & {\left[\begin{array}{cccc}
\mathrm{c} \theta_{i} & -\mathrm{c} \propto_{i} * \mathrm{~s} \theta_{i} & \mathrm{~s} \propto_{i} * \mathrm{~s} \theta_{i} & a_{i} \mathrm{c} \theta_{i} \\
\mathrm{~s} \theta_{i} & \mathrm{c} \propto_{i} * \mathrm{c} \theta_{i} & -\mathrm{s} \propto_{i} * \mathrm{c} \theta_{i} & a_{i} \mathrm{~s} \theta_{i} \\
0 & \mathrm{~s} \propto_{i} & \mathrm{c} \propto_{i} & d_{i} \\
0 & 0 & 0 & 1
\end{array}\right] } \\
& T_{6}^{0}={ }^{0} A_{1}{ }^{1} A_{2}{ }^{2} A_{3}{ }^{3} A_{4}{ }^{4} A_{5}{ }^{5} A_{6}
\end{aligned}
$$




$$
T_{6}^{0}=\left[\begin{array}{cccc}
r_{11} & r_{12} & r_{13} & d_{x} \\
r_{21} & r_{22} & r_{23} & d_{y} \\
r_{31} & r_{32} & r_{33} & d_{z} \\
0 & 0 & 0 & 1
\end{array}\right]
$$

Where:

$$
\begin{aligned}
& r_{11}=-c_{6}\left(c_{5}\left(c_{1} s_{4}-c_{4} s_{1} s_{2}\right)-c_{2} s_{1} s_{5}\right)-s_{6}\left(c_{1} c_{4}+s_{1} s_{2} s_{4}\right) \\
& r_{21}=-c_{6}\left(c_{5}\left(s_{1} s_{4}-c_{1} c_{4} s_{2}\right)-c_{2} s_{1} s_{5}\right)-s_{6}\left(c_{4} s_{1}+c_{1} s_{2} s_{4}\right) \\
& r_{31}=c_{6}\left(s_{2} s_{5}-c_{2} c_{4} c_{5}\right)+c_{2} s_{4} s_{6} \\
& r_{12}=s_{6}\left(c_{5}\left(c_{1} s_{4}-c_{4} s_{1} s_{2}\right)-c_{2} s_{1} s_{5}\right)-c_{6}\left(c_{1} c_{4}+s_{1} s_{2} s_{4}\right) \\
& r_{22}=s_{6}\left(c_{5}\left(s_{1} s_{4}+c_{1} c_{4} s_{2}\right)+c_{1} c_{2} s_{5}\right)-c_{6}\left(c_{4} s_{1}-c_{1} s_{2} s_{4}\right) \\
& r_{32}=c_{2} c_{6} s_{4}-s_{6}\left(s_{2} s_{5}+c_{2} c_{4} c_{5}\right) \\
& r_{13}=-s_{5}\left(c_{1} s_{4}+c_{4} s_{1} s_{2}\right)-c_{2} c_{5} s_{1} \\
& r_{23}=c_{1} c_{2} c_{5}-s_{5}\left(s_{1} s_{4}+c_{1} c_{4} s_{2}\right) \\
& r_{33}=c_{5} s_{2}-c_{2} c_{4} s_{5} \\
& d_{x}=-D_{2} c_{1}-D_{6}\left(s_{5}\left(c_{1} s_{4}-c_{4} s_{1} s_{2}\right)+c_{2} c_{5} s_{1}\right)-D_{3} c_{2} s_{1} \\
& d_{y}=D_{3} c_{1} c_{2}-D_{6}\left(s_{5}\left(s_{1} s_{4}+c_{1} c_{4} s_{2}\right)-c_{1} c_{2} c_{5}\right)-D_{2} s_{1} \\
& d_{z}=D_{1}-D_{6}\left(c_{5} s_{2}+c_{2} c_{4} s_{5}\right)-D_{3} s_{2}
\end{aligned}
$$

\subsection{Robot Stanford with MGC}

This new method, break the schemes of the traditional method of modelling in open kinematic chains, as mentioned the coordinated systems are assigned following the kinematic chain and simply multiplied the matrices in the same order of the movements made, simplifying the mathematics and easing the method. In this demonstration all the movements in whatever direction would be considered, in simple words, including movements in Y axis. Based in the figure 10, that the geometrical schematic representation of the Stanford Robot, and following MGC method to obtain the Transformation matrix. The figure 10 is obtained:

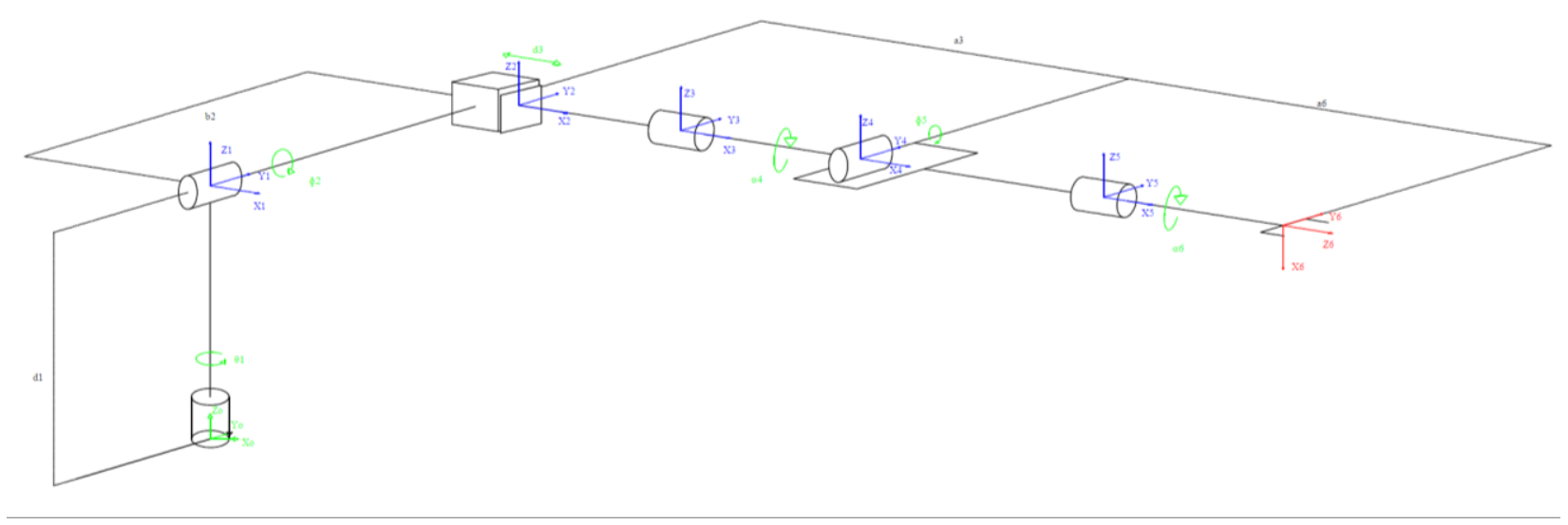

Fig. 10: Movements of the mobile coordinates systems through the Stanford Robot using MGC

Once stablished the coordinates systems, the movements would be presented in table to evaluate all the variables involved and proceed to multiply, and the parameters found during the development based in figure 10 are shown in table 2.

Table 2: MGC parameters of a Stanford robot.

\begin{tabular}{|c|c|c|c|c|c|c|c|c|c|}
\hline Movement & $R_{z, \theta 1}$ & $T_{z, d 1}$ & $R_{y, \phi 2}$ & $T_{y, b 2}$ & $T_{x, a 3}$ & $R_{x, \alpha 4}$ & $R_{y, \phi 5}$ & $R_{y, \alpha 6}$ & $T_{x, a 6}$ \\
\hline value & $\theta_{1}$ & $d_{1}$ & $\phi_{2}$ & $b_{2}$ & $a_{3}$ & $\alpha_{4}$ & $\phi_{5}$ & $\alpha_{6}$ & $a_{6}$ \\
\hline
\end{tabular}


Now applying the matrix post-multiplication principle, to find the homogeneous matrix transformation of the robot equation (16), a multiplication in the same order or the movement needs to be done. It is important to point the fact that $\theta_{2}$, $\phi_{2}, a_{3}, \alpha_{4}, \phi_{5}, \alpha_{6}$ are the six variables of the systems, in other words, the 6 DOF of the Stanford Robot.

$$
H_{6}^{0}=\left[\begin{array}{cccc}
r_{11} & r_{12} & r_{13} & d_{x} \\
r_{21} & r_{22} & r_{23} & d_{y} \\
r_{31} & r_{32} & r_{33} & d_{z} \\
0 & 0 & 0 & 1
\end{array}\right]
$$

Where:

$$
\begin{aligned}
& r_{11}=c_{\phi_{2}} c_{\phi_{5}} c_{\theta_{1}}-s_{\phi_{5}}\left(s_{\alpha_{4}} c_{\theta_{1}}+c_{\alpha_{4}} c_{\theta_{1}} s_{\phi_{2}}\right) \\
& r_{21}=s_{\phi_{5}} s_{\alpha_{4}} c_{\theta_{1}}-c_{\alpha_{4}} s_{\phi_{2}} s_{\theta_{1}}+c_{\phi_{2}} c_{\phi_{5}} s_{\theta_{1}} \\
& r_{31}=-c_{\phi_{5}} s_{\phi_{2}}-c_{\alpha_{4}} c_{\phi_{2}} s_{\phi_{5}} \\
& r_{12}=s_{\alpha_{6}}\left(c_{\phi_{5}}\left(s_{\alpha_{4}} s_{\theta_{1}}-c_{\alpha_{4}} c_{\theta_{1}} s_{\phi_{2}}\right)+c_{\phi_{2}} c_{\theta_{1}} s_{\phi_{5}}\right)-c_{\alpha_{6}}\left(c_{\alpha_{4}} s_{\theta_{1}}-s_{\alpha_{4}} c_{\theta_{1}} s_{\phi_{2}}\right) \\
& r_{22}=-c_{\alpha_{6}}\left(c_{\phi_{5}}\left(s_{\alpha_{4}} c_{\theta_{1}}-c_{\alpha_{4}} s_{\phi_{2}} s_{\theta_{1}}\right)-c_{\phi_{2}} s_{\phi_{5}} s_{\theta_{1}}\right)-s_{\alpha_{6}}\left(c_{\alpha_{4}} c_{\theta_{1}}-s_{\alpha_{4}} s_{\phi_{2}} s_{\theta_{1}}\right) \\
& r_{32}=c_{\alpha_{6}} c_{\phi_{2}} s_{\alpha_{4}}-s_{\alpha_{6}}\left(s_{\phi_{2}} s_{\phi_{5}}-c_{\alpha_{4}} c_{\phi_{2}} c_{\phi_{5}}\right) \\
& r_{13}=s_{\alpha_{6}}\left(c_{\alpha_{4}} s_{\theta_{1}}-s_{\alpha_{4}} c_{\theta_{1}} s_{\phi_{2}}\right)+c_{\alpha_{6}}\left(c_{\phi_{5}}\left(s_{\alpha_{4}} s_{\theta_{1}}-c_{\alpha_{4}} c_{\theta_{1}} s_{\phi_{2}}\right)+c_{\phi_{2}} c_{\theta_{1}} s_{\phi_{5}}\right) \\
& r_{23}=-c_{\alpha_{6}}\left(\left(c_{\phi_{5}} s_{\alpha_{4}} c_{\theta_{1}}-c_{\alpha_{4}} s_{\phi_{2}} s_{\theta_{1}}\right)-c_{\phi_{2}} c_{\phi_{5}} s_{\theta_{1}}\right)-s_{\alpha_{6}}\left(c_{\alpha_{4}} c_{\theta_{1}}+s_{\alpha_{4}} s_{\phi_{2}} s_{\theta_{1}}\right) \\
& r_{33}=-c_{\alpha_{6}}\left(s_{\phi_{2}} s_{\phi_{5}}-c_{\alpha_{4}} c_{\phi_{2}} c_{\phi_{5}}\right)-c_{\phi_{2}} s_{\alpha_{4}} s_{\alpha_{6}} \\
& d_{x}=a_{3} c_{\phi_{2}} c_{\theta_{1}}-b_{2} s_{\theta_{1}}-a_{6}\left(s_{\phi_{5}}\left(s_{\alpha_{4}} c_{\theta_{1}}-c_{\alpha_{4}} c_{\theta_{1}} s_{\phi_{2}}\right)-c_{\phi_{2}} c_{\phi_{5}} c_{\theta_{1}}\right) \\
& d_{y}=b_{2} c_{\theta_{1}}+a_{6}\left(s_{\phi_{5}}\left(s_{\alpha_{4}} c_{\theta_{1}}-c_{\alpha_{4}} s_{\phi_{2}} s_{\theta_{1}}\right)+c_{\phi_{2}} c_{\phi_{5}} s_{\theta_{1}}\right)+a_{3} c_{\phi_{2}} s_{\theta_{1}} \\
& d_{z}=d_{1}-a_{3} s_{\phi_{2}}-a_{6}\left(c_{\phi_{5}} s_{\phi_{2}}+c_{\alpha_{4}} c_{\phi_{2}} s_{\phi_{5}}\right)
\end{aligned}
$$

\begin{tabular}{|c|c|c|}
\hline Parameter & MGC & DHs \\
\hline Steps & Average 6 & Average 14 \\
\hline Restrictions & Follow the kinematic chain & $\begin{array}{l}\text { Only movements in } \mathrm{Z} \text { and then in } \mathrm{X} \\
\text { following the kinematic chain }\end{array}$ \\
\hline Variables & 6 & 6 \\
\hline method & Matrix post-multiplication & Matrix post-multiplication \\
\hline $\begin{array}{l}\text { Final position equations for } \\
\text { the Stanford Robot }(x, y, z)\end{array}$ & $\begin{array}{l}d_{x}=a_{3} c_{\phi_{2}} c_{\theta_{1}}-b_{2} s_{\theta_{1}}-a_{6}\left(s_{\phi_{5}}\left(s_{\alpha_{4}} c_{\theta_{1}}-\right.\right. \\
\left.\left.c_{\alpha_{4}} c_{\theta_{1}} s_{\phi_{2}}\right)-c_{\phi_{2}} c_{\phi_{5}} c_{\theta_{1}}\right) \\
d_{y}=b_{2} c_{\theta_{1}}+a_{6}\left(s_{\phi_{5}}\left(s_{\alpha_{4}} c_{\theta_{1}}-c_{\alpha_{4}} s_{\phi_{2}} s_{\theta_{1}}\right)+\right. \\
\left.c_{\phi_{2}} c_{\phi_{5}} s_{\theta_{1}}\right)+a_{3} c_{\phi_{2}} s_{\theta_{1}} \\
d_{z}=d_{1}-a_{3} s_{\phi_{2}}-a_{6}\left(c_{\phi_{5}} s_{\phi_{2}}+c_{\alpha_{4}} c_{\phi_{2}} s_{\phi_{5}}\right)\end{array}$ & $\begin{array}{l}d_{x}=-D_{2} c_{1}-D_{6}\left(s_{5}\left(c_{1} s_{4}-c_{4} s_{1} s_{2}\right)+\right. \\
\left.c_{2} c_{5} s_{1}\right)-D_{3} c_{2} s_{1} \\
d_{y}=D_{3} c_{1} c_{2}-D_{6}\left(s_{5}\left(s_{1} s_{4}+c_{1} c_{4} s_{2}\right)-\right. \\
\left.c_{1} c_{2} c_{5}\right)-D_{2} s_{1} \\
d_{z}=D_{1}-D_{6}\left(c_{5} s_{2}+c_{2} c_{4} s_{5}\right)-D_{3} s_{2}\end{array}$ \\
\hline $\begin{array}{c}\text { Evaluating, every angle equals } \\
\text { zero }\end{array}$ & $\begin{array}{c}d_{x}=-b 2 \\
d_{y}=a 3+a 6 \\
d_{z}=d 1\end{array}$ & $\begin{array}{c}d_{x}=-D 2 \\
d_{y}=D_{3}+D_{6} \\
d_{y}=D_{1}\end{array}$ \\
\hline
\end{tabular}

\subsection{Comparison between the two methods.}

In this final section, the result of the work is presented facing the two methods in the table 3 and finally making the final quantitative verification, using every angle to be equals zero $\left(0^{\circ}\right)$.

Table 3: MGC vs DHs.

\section{Conclusion}

Assigning coordinates systems is one of the greatest complexities in the DH method, leading to difficulties and errors made in publications, thesis and books. 
The MGC method is easy to understand and apply, avoiding the need to memorize the criteria used in the DH method, the geometrical assumption in some formulas, just obtain the parameters to apply in other little friendly algorithms.

Standard and modified DH methods leads to the same answer, but leads to errors by assigning coordinates systems in one method and using the variables in the other one, producing that the final matrix obtained failed in evaluation.

The final equations validates all the work proposed by the new method and even though the parameters has a different denomination, the geometrical meaning is the same for the two methods based in figure 9 and 10 .

MGC represent a higher level of application than most of the proposed presented before to modelling open kinematic chains, using the same general principle as the one used in DH method.

\section{References}

[1] J. Denavit, R. S. Hartenberg, "A kinematic notation for lower-pair mechanisms based on matrices," ASME Journal of Applied Mechanisms, vol. 23, pp. 215-221, 1955.

[2] P.I. Corke, "A robotics toolbox for MATLAB," Robotics \& Automation Magazine, IEEE, vol. 3(1), pp. 24-32, 1996.

[3] R. W. Brockett, "Robotic manipulators and the product of exponentials formula," in: Fuhrmann, P. (Ed.), Mathematical Theory of Networks and Systems. Springer Berlin / Heidelberg, pp. 120-129, 1984.

[4] Y. Jin and W. Xiaorong, "The application of the dual number methods to scara kinematics," in: International Conference on Mechanic Automation and Control Engineering (MACE), June 2010. pp. 3871-3874.

[5] J. M. McCarthy, "Dual Orthogonal Matrices in Manipulator Kinematics," The International Journal of Robotics Research, vol. 5(2), pp. 45-51, June 1986.

[6] A. Ollero, Robótica: Manipuladores y robots móviles, 1st Edition. MARCOMBO, 2001.

[7] A. Barrientos, L. Peñin, C. Balaguer, R. Aracil, 2007. "Fundamentos de robótica", 2nd Edition. McGraw-Hill.

[8] Paul, R. P., Robot manipulators: mathematics, programming, and control, 1st Edition. The MIT Press, Cambridge, MA, USA, Nov. 1981.

[9] A. Perez and J. M. McCarthy, "Dual Quaternion Synthesis of Constrained Robotic Systems," Journal of Mechanical Design, vol. 126 (3), pp. 425, 2004.

[10] J. Craig, Introduction to Robotics Mechanics and Control, 3rd edition. USA: PEARSON Prentice Hall, 2005.

[11] K. Fu, R. Gonzalez, C. Lee, Robótica: Control, detección, visión e inteligencia, España: Ed. Mc Graw Hill.

[13] J. Shigley and Jr. J. Uicker, Teoría De Maquinas Y Mecanismos, Mexico: Mc Graw Hill, 1988.

[14] M. Spong, S. Hutchinson, and M. Vidyasagar, Robot Dynamics and Control. USA: ED. John Wiley \& Sons, Inc, 2004.

[15] M. Granja, “Comparación entre el método Denavit-Hartenberg estándar y modificado,” ECUADOR: EPN, 2014. 